\title{
Clinical evaluation of ultrasound screening in follow-up visits of infants with cerebral palsy at high risk for developmental dysplasia of the hip
}

\author{
AIZHEN QIU, ZHONGXIU YANG, JIPING WANG and TAOTAO WANG \\ Department of Rehabilitation, Xuzhou Chlidren's Hospital, Xuzhou, Jiangsu 221002, P.R. China
}

Received November 12, 2015; Accepted April 11, 2016

DOI: 10.3892/etm.2016.3653

\begin{abstract}
The aim of the study was to assess the clinical value of ultrasound screenings for the developmental dysplasia of the hip (DDH) and explore its etiology in high-risk infants with cerebral palsy in follow-up visits. A group of 98 cases of infants at high-risk of cerebral palsy who received rehabilitation treatment between July, 2009 and July, 2010 were selected. Infants included 58 men and 40 women, aged $<6$ months and not lost to follow-up visits. Ultrasound (using Graf static inspection) screening of hips was performed and the infants with abnormalities were given clinical intervention, and 1- to 2 -year-old infants were given outpatient follow-ups. The results were analyzed and there were 40 abnormal cases among the 98 cases of infants at high risk of cerebral palsy, including 18 cases of unstable hip joint, and 22 cases of DDH (12 cases of hip dysplasia, 3 cases of hip subluxation and 7 cases of hip dislocation). Early clinical intervention for infants with hip dysplasia and outpatient follow up for infants aged 1-2 years was carried out and had ischemic necrosis of femoral head, with the exception of 1 case of femoral detorsion that was poorly restored. In conclusion, the probability of DDH was higher in infants at high-risk of cerebral palsy compared to the normal infants. Hip ultrasound is a safe, simple, and effective screening method for these infants, which is of great clinical significance for an earlier diagnosis and treatment of DDH in infants with cerebral palsy.
\end{abstract}

\section{Introduction}

Developmental dysplasia of the hip (DDH) is one of the most common diseases in the skeletal system of infants as well as the primary cause of degenerative hip arthritis in adults $(1,2)$. There is no complete DDH statistical material available in

\footnotetext{
Correspondence to: Dr Zhongxiu Yang, Department of Rehabilitation, Xuzhou Chlidren's Hospital, 18 Sudibei Road, Xuzhou, Jiangsu 221002, P.R. China

E-mail: yangzhongxiu21@163.com
}

Key words: developmental dysplasia of the hip, infants at high-risk of cerebral palsy, ultrasonic inspection
China and DDH morbidity is not the same in various regions being lower in the south but higher in the north. According to Chen et al (3), the DDH morbidity in newborns in Shanghai is $3.37 \%$. As Xuzhou is in the north, the DDH morbidity statistics of infants as reported by ultrasound room in Xuzhou Children's Hospital was higher than that of Shanghai, while the difference for premature infants was more significant (4). The curative effect of DDH depends on early diagnosis and treatment; clinical and physical examination, as well as X-ray and magnetic resonance (MR) examinations were applied to make the diagnosis (5-8). As for newborns and infants aged $<6$ months, the false-negative results of clinical and physical examinations were higher. Children undergoing treatment are exposed to the damages of ionizing radiation several times during X-ray examination and also have to remain under sedation for a long period of time when undergoing MR examination (9-12). These tests are expensive and not suitable for general examination of infants. In recent years, ultrasonic testing has become increasingly popular, enabling DDH infants to undergo early diagnosis and treatment, thereby lowering the occurrence rate at a later stage. At present, whether it is appropriate to conduct extensive general examination of DDH infants remains to be determined, but it is important to conduct general investigation of high-risk DDH infants (13-15).

In the present study, we assessed the clinical value of ultrasound screenings for the developmental dysplasia of the hip (DDH) and explore its etiology in high-risk infants with cerebral palsy in follow-up visits. The Department of Rehabilitation at the Xuzhou Children's Hospital (Jiangsu, China) observed preferable effect of ultrasound screening for $\mathrm{DDH}$ in order to reduce the disability rate in high-risk infants with cerebral palsy in follow-up visits and early intervention treatment for abnormal cases.

\section{Subjects and methods}

Subjects. A total of 146 high-risk infants with cerebral palsy, aged $<6$ months and who had received rehabilitation treatment at the Xuzhou Children's Hospital between July, 2009 and July, 2010 were selected for the present study. Of the 146 cases, 48 cases were lost to follow up while the remaining 98 cases were included for periodic outpatient follow-up and a file for high-risk infants with cerebral palsy was created. The criteria used to evaluate a high-risk infant with cerebral palsy were: 
Table I. Inspection results of hip ultrasonic test on 98 high-risk infants with cerebral palsy.

\begin{tabular}{lcccc}
\hline Inspection results & Cases & Bone apical line angle $\alpha$ & Cartilage apical line angle $\beta$ & Femoral head coverage rate MRI \\
\hline Normal hip joint & 58 & $\alpha>60^{\circ}$ & $\beta<55^{\circ}$ & $55 \%<\mathrm{MRI}<60 \%$ \\
Unstable hip joint & 18 & $55^{\circ}<\alpha<60^{\circ}$ & $55^{\circ}<\beta<77^{\circ}$ & $45 \%<\mathrm{MRI}<55 \%$ \\
Hip dysplasia & 12 & $50^{\circ}<\alpha<55^{\circ}$ & $55^{\circ}<\beta<77^{\circ}$ & $45 \%<\mathrm{MRI}<55 \%$ \\
Hip subluxation & 3 & $45^{\circ}<\alpha<50^{\circ}$ & $\beta>77^{\circ}$ & $\mathrm{MRI}<45 \%$ \\
Hip dislocation & 7 & $\alpha<45^{\circ}$ & Unmeasured & Unmeasured \\
\hline
\end{tabular}

MRI, magnetic resonance imaging.

Table II. Proportions of male and female high-risk infants with cerebral palsy and DDH.

\begin{tabular}{lccccc}
\hline Gender & Normal hip joint & Unstable hip joint & Hip dysplasia & Hip subluxation & Hip dislocation \\
\hline Male & 33 & 14 & 7 & 1 & 3 \\
Female & 25 & 4 & 5 & 2 & 4 \\
\hline
\end{tabular}

DDH, developmental dysplasia of the hip.

i) Infants with high-risk factors including hypoxic ischemic encephalopathy, gastrin with low birth weight, birth asphyxia, intracranial hemorrhage, intrauterine growth retardation, intrauterine infection, polyhydramnios or oligoamniosan pathologic jaundice; and ii) infants who get abnormal results for qualitative general movements assessment.

Ultrasonic testing. An ultrasonic diagnostic equipment (Siemens, Nuremberg, Germany) with linear transducer frequency of $6.5 \mathrm{MHZ}$ was used in the present study. According to the Graf static test, coronal plane graphs of hip joints in the femur trochanter were obtained when the infants were tested in the lateral position. The standard images were those with straight ilium as well as openings and echoes in the inner edge of acetabular cartilage and echoes in the outer acetabular labrum simultaneously. The images contained the junction of complete femoral head and upper cacific femur. The major parameters of testing were bone apical line angle $\alpha$ and cartilage apical line angle $\beta$. Femoral head coverage rate MR imaging (MRI) was also tested.

Standard of classification. The status of hip was classified as: Normal hip joint: $\alpha \geq 60^{\circ}, \beta<55^{\circ}, 55 \% \leq \mathrm{MRI}<60 \%$; unstable hip joint: $55^{\circ} \leq \alpha<60^{\circ}, 55^{\circ}<\beta \leq 77^{\circ}, 45 \% \leq \mathrm{MRI}<55 \%$; hip dysplasia: $50^{\circ} \leq \alpha<55^{\circ}, 55^{\circ}<\beta \leq 77^{\circ}, 45 \% \leq \mathrm{MRI}<55 \%$; hip subluxation: $45^{\circ} \leq \alpha<50^{\circ}, \beta>77^{\circ}, \mathrm{MRI}<45 \%$; hip dislocation: $\alpha<45^{\circ}$. However, $\beta$ and MRI could not be measured.

Clinical diagnosis. The clinical diagnosis was mainly determined by ultrasonic inspection results. According to the Graf classification method, the hip joints were divided into: Normal hip joint, unstable hip joint, dislocation of hip joint (including hip displasia, hip subluxation and hip dislocation).

Early intervention measures. Early intervention measures included: i) Communication with the rehabilitation therapists to avoid exacerbating dislocation of hip joint by pulling the hip joint and placing a heavy load on the lower limb; ii) the hip joints of infants aged $<3$ months in abduction and external rotation were kept by 'wide diaper' method, parents were instructed to avoid using 'baby bunting' and to do hip flexor abduction exercises until the infant patients underwent re-examination of ultrasonic inspection at 3 months of age, and the subsequent therapeutic schedule was decided; iii) 'wide diaper' method was continued for 3- to 5-month-old infants with unstable hip joint, while Pavlik sling treatment was employed for infants with hip displasia, subluxation and dislocation.

Follow-up visit. During early intervention treatment, ultrasonic inspection was conducted once every four weeks until two successive re-examinations showed normal hip joint. A pelvic X-ray examination was used to inspect the infants at $>1$ year of age.

\section{Results}

The results showed that of 98 cases of high-risk infants with cerebral palsy, 40 cases had abnormal hip joint, including 18 cases of unstable hip joint, and $22 \mathrm{DDH}$ cases (12 cases of hip dysplasia, 3 cases of hip subluxation, 7 cases of hip dislocation). Infants with hip dysplasia and DDH accounted for 40.8 and $22.4 \%$, respectively (Table I). For the 11 cases of unstable hip joint, 7 were unilateral unstable hip joint cases ( 5 left, 2 right); of the hip dysplasia patients, 3 were bilateral ones, 5 left and 2 right, and 2 cases had dyplasia on the left hip joint and 1 case had instability on the right; there were 2 cases of left hip subluxation, 1 case of right hip subluxation with unstable left hip joint among infants with hip subluxation; there were 3 cases of bilateral hip dislocation, 2 cases of left hip dislocation, 1 case of right hip dislocation and 1 case of right hip dislocation with left hip dysplasia in all the hip dislocation cases. There were 25 men (accounting 
for $43.1 \%$ of male high-risk infants with cerebral palsy) and 15 women (accounting for $37.5 \%$ of female high-risk infants with cerebral palsy) who had DDH (Table II).

Following early intervention treatment for high-risk infants with cerebral palsy and DDH, infants who previously had unstable hip joint were returned to normal according to the ultrasonic re-examination 3 months later at follow up. There were no case of ischemic necrosis of femoral head in any of the DDH infants aged 1-2 years in the follow-up visit while only 1 case of femoral detorsion was poorly restored and needed continuous treatment at the Department of Orthopaedics.

\section{Discussion}

The morbidity rate of DDH is approximately $1 \%$. When an infant is born with hip dysplasia, the acetabulum and head of femur lack appropriate development $(16,17)$. If the dislocation is not restored in a timely manner, dysplasia of the hip is exacerbated with age. By contrast, if the dislocation is restored in a few days or weeks after birth, dysplasia of the hip can be reversed and normal development achieved. As the infant develops, and starts walking, joint deformity gradually becomes aggravated and is not restored permanently if the dislocation and subluxation are not appropriately treated (18). Therefore early diagnosis and treatment are important (19).

Ultrasonic inspection, with its strong sensibility, zero ionizing radiation damage and high reproducibility, can be used to assess the developmental state of bone, cartilage and soft tissue in articulars and show the early anatomical structure of hip joints $(20,21)$. Professor Graf, the founder of hip ultrasound screening and Austrian specialist of orthopedics, applied ultrasound for the inspection of hip joints of infants in the 1970s, a method now employed worldwide (22). DDH morbidity in previous reports (23) ranged from 3.75 to $109 \%$, but the results of ultrasound screening were always higher, which may be related to the timing of screening and standard of diagnosis as well as the subjectivity and proficiency of the scrutators. At present, whether it is appropriate to conduct extensive general investigation for DDH in infants remains to be determined. However, we considered it necessary to conduct general investigation of DDH in high-risk infants as its morbidity is on the increase (24-26).

The reasons for high-risk infants with cerebral palsy having DDH are: i) The pathogenesis of cerebral palsy is widely considered to be the result of the early development of the embryo. Many infants with cerebral palsy are born with congenital deformity while DDH is also caused by developmental factors; ii) high-risk infants with cerebral palsy have abnormal muscular tension, especially the tension of adductor, which also affects the development of hip joints $(27,28)$. The occurence rate of high-risk infants with cerebral palsy having DDH is high, but the clinical symptoms are always neglected as the abnormal muscular tension and muscle strength occur extremely early. Therefore, by the time parents discover that their children walk abnormally, the optimal time for treatment has been missed (29). As children become older, the treatment becomes more difficult and surgery is required, resulting in increasing amounts of pain for infants and the family as well as an increase of the burden on society. Therefore, it is of great clinical significance to conduct DDH ultrasound screening and early intervention treatment for high-risk infants with cerebral palsy.

\section{References}

1. Rosenberg N, Bialik V, Norman D and Blazer S: The importance of combined clinical and sonographic examination of instability of the neonatal hip. Int Orthop 22: 185-188, 1998.

2. Afaq AA, Stokes S, Fareed H, Zadeh HG and Watson M: Ultrasound in the selective screening of developmental dysplasia of the hip. Eur Rev Med Pharmacol Sci 15: 394-398, 2011.

3. Chen BC, Yang J, Hong W, et al: DDH ultrasound screening for newborns. Chin J Pediatr Surg 28: 590-593, 2007.

4. Chen BC: Developmental abnormalities of hip ultrasound examination. Clin J Pediatr Surg 2: 196-200, 2003.

5. Ponce de León Samper MC, Herrera Ortiz G and Castellanos Mendoza C: Relationship between flexible flat foot and developmental hip dysplasia. Rev Esp Cir Ortop Traumatol 59: 295-298, 2015.

6. Huayamave V, Rose C, Serra S, Jones B, Divo E, Moslehy F, Kassab AJ and Price CT: A patient-specific model of the biomechanics of hip reduction for neonatal Developmental Dysplasia of the Hip: Investigation of strategies for low to severe grades of Developmental Dysplasia of the Hip. J Biomech 48: 2026-2033, 2015.

7. Jackson JC, Runge MM and Nye NS: Common questions about developmental dysplasia of the hip. Am Fam Physician 90: 843-850, 2014.

8. Bin K, Laville JM and Salmeron F: Developmental dysplasia of the hip in neonates: Evolution of acetabular dysplasia after hip stabilization by brief Pavlik harness treatment. Orthop Traumatol Surg Res 100: 357-361, 2014.

9. Sakellariou VI, Christodoulou M, Sasalos G and Babis GC: Reconstruction of the acetabulum in developmental dysplasia of the hip in total hip replacement. Arch Bone Jt Surg 2: 130-136, 2014.

10. Binazzi R: Two-stage progressive femoral lowering followed by cementless total hip arthroplasty for treating Crowe IVHartofilakidis type 3 developmental dysplasia of the hip. J Arthroplasty 30: 790-796, 2015.

11. Bilgen ÖF, Salar N, Bilgen MS, Mutlu M, Kara GK and Gürsel E: The effect of dislocation type (crowe types i-iv) on pelvic development in developmental dysplasia of the hip: a radiologic study of anatomy. J Arthroplasty 30: 875-878, 2015.

12. Kaneko H, Kitoh H, Mishima K, Matsushita M, Kadono I, Ishiguro $\mathrm{N}$ and Hattori T: Factors associated with an unfavourable outcome after Salter innominate osteotomy in patients with unilateral developmental dysplasia of the hip: Does occult dysplasia of the contralateral hip affect the outcome? Bone Joint J 96-B: 1419-1423, 2014.

13. Schwend RM, Shaw BA and Segal LS: Evaluation and treatment of developmental hip dysplasia in the newborn and infant. Pediatr Clin North Am 61: 1095-1107, 2014.

14. Ike H, Inaba Y, Kobayashi N, Yukizawa Y, Hirata Y, Tomioka M and Saito T: Effects of rotational acetabular osteotomy on the mechanical stress within the hip joint in patients with developmental dysplasia of the hip: A subject-specific finite element analysis. Bone Joint J 97-B: 492-497, 2015.

15. Ramani N, Patil MS and Mahna M: Outcome of surgical management of developmental dysplasia of hip in children between 18 and 24 months. Indian J Orthop 48: 458-462, 2014.

16. Teixeira SR, Dalto VF, Maranho DA, Zoghbi-Neto OS, Volpon JB and Nogueira-Barbosa MH: Comparison between Graf method and pubo-femoral distance in neutral and flexion positions to diagnose developmental dysplasia of the hip. Eur J Radiol 84: 301-306, 2015.

17. Inoue D, Kabata T, Maeda T, Kajino Y, Fujita K, Hasegawa K, Yamamoto $\mathrm{T}$ and Tsuchiya $\mathrm{H}$ : Value of computed tomography-based three-dimensional surgical preoperative planning software in total hip arthroplasty with developmental dysplasia of the hip. J Orthop Sci 20: 340-346, 2015.

18. Carlile GS, Woodacre T and Cox PJ: Verification of hip reduction using anterior ultrasound scanning during Pavlik harness treatment of developmental dysplasia of the hip. J Orthop 11: 174-179, 2014.

19. Qin M, Du JX, Wang QS, et al: Ultrasonic inspection for DDH premature infants. J Bengbu Med Coll 35: 404-406, 2010. 
20. Cavuto A, Martarelli M, Pandarese G, Revel GM and Tomasini EP: Experimental investigation by laser ultrasonics for high speed train axle diagnostics. Ultrasonics 55: 48-57, 2015.

21. Rupin F, Blatman G, Lacaze S, Fouquet T and Chassignole B: Probabilistic approaches to compute uncertainty intervals and sensitivity factors of ultrasonic simulations of a weld inspection. Ultrasonics 54: 1037-1046, 2014.

22. Graf R: New possibilities for the diagnosis of congenital hip joint dislocation by ultrasonography. J Pediatr Orthop 3: 354-359, 1983.

23. Gala L, Clohisy JC and Beaulé PE: Hip dysplasia in the young adult. J Bone Joint Surg Am Jan 98: 63-73, 2016.

24. Imbuldeniya AM, Walter WL, Zicat BA and Walter WK: Cementless total hip replacement without femoral osteotomy in patients with severe developmental dysplasia of the hip Minimum 15-year clinical and radiological results. Bone Joint J 96-B: 1449-1454, 2014.

25. Chen KH, Wang HY, Sung CS, Wu PK, Chen CF, Liu CL and Chen WM: Wake-up test in total hip arthroplasty with high-riding developmental dysplasia. Orthopedics 37: e625-e630, 2014
26. Starr V and Ha BY: Imaging update on developmental dysplasia of the hip with the role of MRI. AJR Am J Roentgenol 203: 1324-1335, 2014

27. Carroll KL, Schiffern AN, Murray KA, Stevenson DA, Viskochil DH, Toydemir R, MacWilliams BA and Roach JW: The occurrence of occult acetabular dysplasia in relatives of individuals with developmental dysplasia of the hip. J Pediatr Orthop 1, 2015

28. Yoon PW, Kim JI, Kim DO, Yu CH, Yoo JJ, Kim HJ and Yoon KS: Cementless total hip arthroplasty for patients with Crowe type III or IV developmental dysplasia of the hip: two-stage total hip arthroplasty following skeletal traction after soft tissue release for irreducible hips. Clin Orthop Surg 5: 167-173, 2013.

29. Wei KL: Modern Diagnosis and Treatment of Pediatric Diseases. People's Medical Publishing House, Beijing, pp809-811, 1998 (In Chinese). 